Short Communication

\title{
EFFECTIVENESS OF STRUCTURED ACTIVITY PROGRAM ME ON COGNITIVE FUNCTION AMONG INMATES OF THE DESTITUTE HOME
}

\begin{abstract}
Anjali K.G. ${ }^{1}$, Asha K. Nayak ${ }^{2} \&$ Maxie Andrade ${ }^{3}$
${ }^{1}$ Lecturer, Department of Psychiatric Nursing, ${ }^{2,3}$ Assistant Professors, Department of Mental Health Nursing, ${ }^{3}$ Department of Community Health Nursing, Manipal College of Nursing M anipal, M anipal University, Manipal.

Correspondence

Anjali K.G.

Lecturer, Department of Psychiatric Nursing, M anipal College of Nursing, M anipal University, M anipal - 576 104, Karnataka, India. Mobile : +919035136877 E-mail : anjubluerose@gmail.com

Abstract:

Current trends in health care practice emphasis on cost effective consolidative approach of pharmacological and life style modification. This can help mentally challenged individual and destitute to obtain the optimum level of functioning. The study aimed to determine the effectiveness of structured activity programme on cognitive function, self-esteem and behaviour among mentally ill destitute home inmates. Study was conceptualized based on Albert Bandura's Triadic reciprocity of determinism. An evaluative approach using pre-test post-test design was used for the study. The samples were selected based on the sampling criteria and a total of 50 samples were recruited for the study with the mean age of $41.98 \pm 13$.13. Study findings revealed that structured activity programme was effective in improving the cognitive function of mentally ill destitute. This suggests structured activities can be used in institutionalized or hospital setting to improve the cognition of mentally ill clients.
\end{abstract}

Keywords : Exercise programme, activity, cognitive function, mentally ill, destitute.

\section{Introduction :}

Destitute is a person who owns no cattle, or substantial amounts of small stock, fields, who have no job, and are not living with close relatives (spouse, parent, or child) who have a source of income. Destitute homes are places where destitute are provided with shelter and food. ${ }^{1}$ The publication of Health Initiative group for homeless in India on Health care beyond Zero: Ensuring a basic right for the homeless reveals that among the mentally ill homeless, around $82 \%$ are chronically mentally ill and $14 \%$ are severely mentally ill. ${ }^{2}$ This findings recommend a cost effective structured activity programme in order to improve the mental health of destitute. Systematic review shows that physical activity has got significant effect on

\begin{tabular}{|c|}
\hline Access this article online \\
\hline Quick Response Code \\
\hline
\end{tabular}

cognitive function, depression, anxiety, stress, subjective wellbeing, emotion and mood. Purpose of the study was to promote prudent living skills among inmates of destitute home through structured activity programme.

\section{Materials and methods:}

Participants: Participants of the study were recruited from selected destitute home, Udupi after obtaining the administrative permissions. Ethical clearance was obtained from institutional ethics committee. Fifty inmates were selected based on the criteria such as who were free from any physical disability and imposed mobility restriction, able to follow instructions and able to comprehend languages like Kannada, English, Hindi and Malayalam. Among the participants males and females were equally distributed. M ean age of the samples was $41.98 \pm 13.13$.

Measures: On the day of pre-test Demographic proforma, personal history was collected from patient records and M ini Mental Status Examination was done by interview method. Demographic variables included were age, gender, education, marital status and state of origin. Psychiatric diagnosis, length of stay in destitute home and duration of treatment was included in personal history. M ini Mental Status examination is a standardized tool to 
assess cognitive function which has got a minimum score of 0 and maximum score of 30 .

\section{Structured activity programme:}

Structured activity programme includes participation in the structured exercise programme and paper bag making activity which is carried out at the designated setting in the destitute home.

Structured exercise therapy means structured movement like flexion, extension, abduction and adduction of various joints of body like neck, shoulder, elbow, wrist, hip, knee, ankle and breathing exercises including brain gym exercise such as yawning up on researcher's instruction and care takers supervision for a group of inmates of destitute home for 30 minutes.

Paper bag making includes making paper bag of a size of 16 $\mathrm{cm}$ length and $12 \mathrm{~cm}$ width. A newspaper of $28 \mathrm{~cm}$ length and $18 \mathrm{~cm}$ width is provided to inmates which to be folded length wise one edge over the other one and after that one end is folded width wise. All the folded areas are pasted with gum to make paper bag. This was taught by the researcher and care takers on the first day. For one month inmates of destitute home performed paper bag making independently under the guidance of care takers for 1 hour 30 minutes every day.

\section{Method:}

After collecting data on demographic proforma and personal history, pre-test cognitive function scores were obtained by interview method. Both males and females were divided in to two groups: 12 members in one group and 13 members in other group based on the code number given to them because of lack of facilities for making all participants to do exercise at same time. All groups were provided with structured activity programme for 5 consecutive days in a week for 4 weeks in the morning. Attendance was taken on each day before the intervention and every session was having 25 minute exercise and 5 minute rest followed by paper bag making for one and half hour which was supervised by four care givers. Rapport with the inmates was made with the help of care takers and during the pre-test. Inmates were placed in straight line for exercise. For the activity both the males and females were made to sit in round group. Paper of size $28 \mathrm{~cm}$ length and $18 \mathrm{~cm}$ width was given to the inmates. With those cut papers of same size and gum they made paper bag of $16 \mathrm{~cm}$ length and $11 \mathrm{~cm}$ width approximately. Everyday number of paper bags made by each inmate was counted by both the inmate and researcher and was recorded. Post-test was done after 3 days of completing 4 weeks intervention programme.

\section{Results:}

\section{Sample characteristics of inmates of destitute home}

Table 1 reveals that

i. Cognitive function was high among people less than 40 years than people aged above 40 years.

ii. M ales had a higher cognitive function than females.

iii. Higher cognitive function was seen among unmarried people.

iv. Inmates staying in the institution for less than 1 year had higher self-esteem than people who stay there from 6 10 years. But among people staying more than 10 years in destitute home had an improved self-esteem.

Sample characteristics of care takers: There were 4 care takers in the institution one male and three females. All were licensed registered nurses and one of the female nurses had the qualification of BSC (N). All were having a minimum work experience of 1 year in destitute home and male nurse had additional 3 years of working experience in general hospital other than one year experience in destitutehome.

\section{Effectiveness of structured activity programme}

Effectiveness of structured activity programme was analysed using Wilcoxon signed rank test as the data was not following normal distribution and found that there is a significant difference between median pre-test and post test scores of cognitive function.( Table 2). Table 2 reveals that structured exercise programme is effective in improving cognitive function. 


\section{Description of Cognitive function scores}

Fig 1.shows that 15 members those who had severe cognitive impairment, after the intervention improved to mild cognitive impairment and other 7 members those who had mild cognitive impairment improved with intervention to no cognitive impairment.

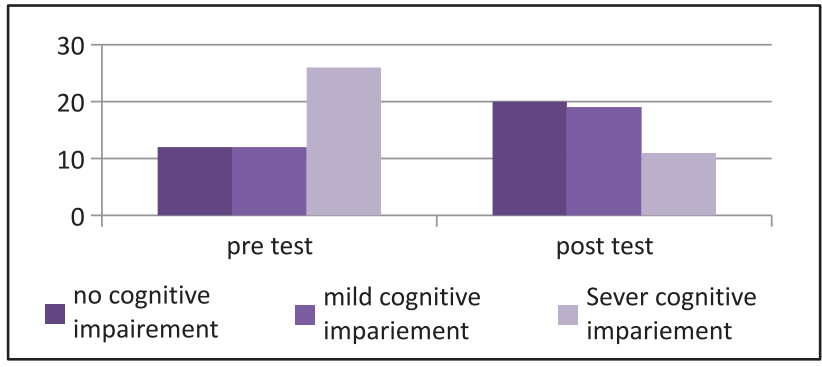

\section{SI No Sample characteristics}

1

Age in years

$\leqslant 40$

$>40$

2

\section{Gender}

Male

Female

3 Marital status

Single

\section{Mean(Standard} deviation)/frequency

41.98

$31.46^{*}$

$53.38^{*}$

(8.722)

25

25

20 $n=50$

function (standard

deviation)

18.06 (5.008)

18.50

(5.361)

(4.662)

$18.52(5.067)$

$17.60(5.008)$ 
Table 2: M edian, Inter Quartile Range(IQR), Wilcoxon Signed Rank test value and $p$ value of pre test and post test cognitive function scores of inmates of destitute home.

$n=50$

\begin{tabular}{lcccc}
\hline & Median & IQR & Z Value & p-value \\
\hline M M SE pretest score & 17 & 8.75 & & \\
& & & 6.051 & $.001^{*}$ \\
M M SE post test score & 21.60 & 7.25 & & \\
\hline
\end{tabular}

$* p<0.05$

\section{Discussion:}

Asha D S in her review done on animal studies summarized that aging of the brain can be prevented by regular exercise. Reviewer says as the studies in human beings on exercise and aging of brain based on neuro chemical changes is difficult animal studies provides base for this knowledge. According to the reviewer exercise regulate protein synthesis; this increases cellular ability to remove damaged proteins by free radicals. Exercise also reduces the brain tissue loss in humans. Regular exercise increases the capillarization, reduced oxidative damage and improvement in memory. This study emphasises the fact that regular exercise can reduce aging of the brain and cognitive impairment. ${ }^{4}$ This finding supported the research finding that exercise can promote cognitive function. Findings of this study are supported by study conducted by Hoffman $\mathrm{H}$ et.al on finding the outcome in schizophrenia outpatients attending vocational rehabilitation programme. Fifty three Schizophrenia clients attending vocational training programmes in Switzerland participated in the study. Among the participants 35 had

\section{References:}

1. Howell N. Demography of the Dobe Kung. $2^{\text {nd }}$ Ed. New Jersey: Transaction publishers; 2009.

2. Desai N G. Health care beyond zero Ensuring a basic right for the homeless: New Delhi: 2003.

3. FoxR K. The influence of physical activity on mental well-being. Public Health Nutrition: 1999; 2(3a), 411.

4. Asha D S. Aging brain: Prevention of oxidative stress by Vitamin E and Exercise. The scientific world journal: 2009; 9: 366.

5. Hoffman H, Kupper Z, Zbinden M , Hirsbrunner H. Predicting vocational functioning and outcome in schizophrenia outpatients attending a vocational rehabilitation programme. Social psychiatry psychiatric epidemiology: 2003; 38: 77. completed the vocational programmes. After three months the cognitive functioning assessment of those clients were done and found a significant improvement (chi square $=9.7 ; p \varangle 0.05$ ). This concludes that Vocational rehabilitation programmes have an effect on Cognitive function. ${ }^{5}$

This emphasises that cost effective structured activity programme can be used for improving cognitive function of institutionalized and hospitalized mentally ill patients.

\section{Conclusion:}

Strengths: Structured activity programme is a cost effective programme which can be used for institutionalized mentally ill for improvement of cognitive function. Study was conducted among destitute home inmates in whom effectiveness of such interventions are not explored.

Limitations: Participants were divided into groups and while one group was doing exercise other group was doing cover making and alternatively. M anpower assistance was used in giving intervention. Focused attention on one to one basis was not able to provide in intervention.

\section{Acknowledgement:}

I express my sincere thanks and deep sense of gratitude to all the participants of this study, administrators for granting permission, validators of the tool and to all those who directly or indirectly helped me during the study. 